\title{
Contact printed masks for 3D microfabrication in negative resists
}

\author{
Häfliger, Daniel; Boisen, Anja
}

Published in:

18th IEEE International Conference on Micro Electro Mechanical Systems, 2005.

Link to article, DOI:

10.1109/MEMSYS.2005.1453990

Publication date:

2005

Document Version

Publisher's PDF, also known as Version of record

Link back to DTU Orbit

Citation (APA):

Häfliger, D., \& Boisen, A. (2005). Contact printed masks for 3D microfabrication in negative resists. In 18th IEEE International Conference on Micro Electro Mechanical Systems, 2005. IEEE.

https://doi.org/10.1109/MEMSYS.2005.1453990

\section{General rights}

Copyright and moral rights for the publications made accessible in the public portal are retained by the authors and/or other copyright owners and it is a condition of accessing publications that users recognise and abide by the legal requirements associated with these rights.

- Users may download and print one copy of any publication from the public portal for the purpose of private study or research.

- You may not further distribute the material or use it for any profit-making activity or commercial gain

- You may freely distribute the URL identifying the publication in the public portal

If you believe that this document breaches copyright please contact us providing details, and we will remove access to the work immediately and investigate your claim. 


\title{
CONTACT PRINTED MASKS FOR 3D MICROFABRICATION IN NEGATIVE RESISTS
}

\author{
D. Haefliger and A. Boisen \\ MIC - Department of Micro- and Nanotechnology, Technical University of Denmark, \\ DTU-bldg. 345 east, DK-2800 Kongens Lyngby, Denmark
}

\begin{abstract}
We present a process based on contact printed shadow masks for three-dimensional microfabrication of soft and sensitive overhanging membranes in SU-8. A metal mask is transferred onto unexposed SU-8 from an elastomer stamp made of polydimethylsiloxane. This mask is subsequently embedded into the negative resist to protect buried material from UV-exposure. Unlike direct evaporation-deposition of a mask onto the SU-8, printing avoids high stress and radiation, thus preventing resist wrinkling and prepolymerization. We demonstrate effective monolithic fabrication of soft, $4-\mu \mathrm{m}$-thick and $100-\mu \mathrm{m}$-long cantilevers integrated in a microfluidic system. The process yields very flat and well-defined membrane surfaces.
\end{abstract}

\section{INTRODUCTION}

Microfabricated structures are increasingly coupled to biology and medicine to produce analytical systems for biochemical and medical diagnostics. Such lab-on-a-chip devices are usually composed of a microfluidic handling system, which can incorporate complex mechanical structures such as valves and pumps. Vital to the fabrication of these components is the ability to three-dimensional structuring. In recent years SU-8, an epoxy-based negative UV-sensitive photoresist [1], has received increasing attention in microfabrication due to its out-standing properties in thick-film processing at high aspect ratio. Unilike the traditionally used silicon and glass, SU-8 is a low-cost material and exhibits high biocompatibility. The polymer is micromachined by cheap and simple spin-coating and UV lithography techniques allowing for short fabrication times and high flexibility in device prototyping. Fully three-dimensional microfabrication in SU-8 is achieved by staking of individual polymer layers.

We present here a novel process for three-dimensional, monolithic fabrication of very thin and soft membranes in SU-8. This work was motivated by our research in cantilevered biosensors. Such biosensors provide information on biochemical reactions on the molecular scale. They monitor changes in surface stress provoked by e.g. antibody-antigen binding or DNA hybridization occurring on the cantilever. Crucial to these devices is a very soft cantilever for high stress sensitivity. Initially fabricated in silicon and silicon nitride [2] we recently manufactured such biosensors in SU8 with full integration in a microfluidic network [3]. SU-8 has a 40 times lower Young's modulus than silicon, which can potentially increase the sensor sensitivity due to the softer material. Negative resists such as SU-8, however, impose an inherent difficulty in fabrication as illustrated in Fig. 1. Fig. 1(a) shows a cross-sectional view of a cantilever overhanging a microfluidic channel. The manufacturing of the cantilever requires the exposure of only a thin layer on the film surface while leaving the bulk material unaffected by radiation. Due to the low absorption of SU-8 in the nearUV region this task becomes rather challenging when employing standard UV lithography. The problem can be circumvented by composing structures as shown in Fig. 1(a) via time-consuming and cumbersome bonding of two chips [3]. Here, we suggest the use of contact printed embedded masks to protect buried resist from UV-exposure [Fig. 1(b)] offering a simpler and faster way to fabricate particularly thin $(<5-\mu \mathrm{m}$-thick) and sensitive overhanging structures. Such embedded shadow masks have been used earlier; however, they were directly evaporated onto the soft, unexposed SU-8 [1]. By using this technique we observed warping of the resist after metal deposition in an electron beam evaporation device (LAB 500, Leybold Optics, Alzenau, Germany). The polymer deformation is supposed to origin from interfacial stress between the metal and polymer provoked by thermal mismatch of the two materials. Moreover, polymerization of the SU-8 due to UV-light and scattered electrons generated during the evaporation process was observed. While warping and pre-polymerization of the SU8 is negligible when working with thick ( $>50 \mu \mathrm{m}$ ) resists [1] it proved to be fatal for very thin membranes [3]. Other techniques such as proton beam exposure [4], UV dosage control [5] and laser writing [6,7] are either expensive or produce stiff membranes of several tens of mictometers thickness. The use of sacrificial layers [8] produces edges in thin SU-8 films, which can cause cracks in the polymer and lead to bad step coverage in metal wires on top of the structure. In the following, a protocol for contact printing of

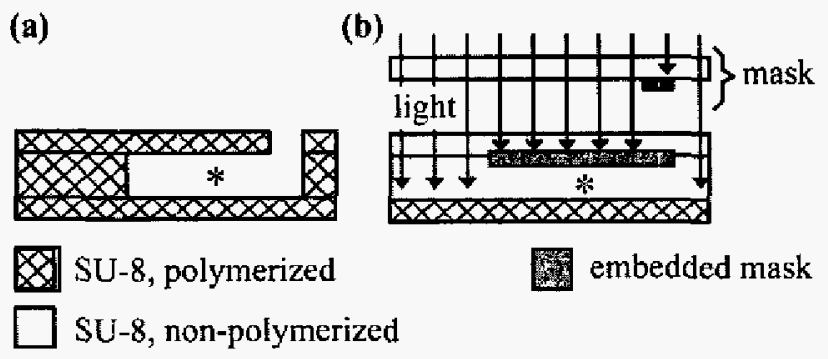

Figure 1: (a) Difficulty in monolithic fabrication of overhanging structures in negative resist: selective polymerization of resist over non-polymerized (*) material. (b) Principle of embedded mask to protect buried resist $\left({ }^{*}\right)$ from $U V$ induced polymerization. 
embedded masks is provided. The process minimizes plastic deformation of the resist material by avoiding direct evaporation-deposition of the metal mask onto the resist. The fabrication of well-defined, soft and sensitive cantilevers integrated in a microfluidic handling system as illustrated in Fig. 1(a) is demonstrated.

\section{FABRICATION PROCESS}

\section{Stamp}

The process sequence for the fabrication of the overhanging cantilevers is schematically illustrated in Fig. 2. The central step in the protocol consists of contact transfer printing [9] of the embedded mask from a soft stamp made of polydimethylsiloxane (PDMS) onto the SU-8 resist [Fig. 2(a)]. The high mechanical flexibility of the elastomer stamp ensures conformal, intimate physical contact between the tool and the substrate on wafer scale. The stamp was molded against a negative master consisting of a $17-\mu \mathrm{m}$ thick structured SU-8 film on a silicon wafer. The liquid prepolymer (Sylgard 184, Dow Corning Corp., Midland, MI, USA) was first poured onto the master and then cured in an oven at $80^{\circ} \mathrm{C}$ for $4 \mathrm{~h}$. After curing the PDMS the stamp was peeled off the mold. It should be noted that the elastomer shrinks about $1 \%$ after its release. This shrinkage needs to be taken into account when fabricating the master to ensure proper alignment between the mask to be stamped and components on the SU-8 microsystem.

Finally, a 50 -nm-thick layer of gold followed by a 20 nm-thick chromium film was evaporation-deposited on the stamp without any surface pretreatment. This metal bilayer formed the shadow mask to be transferred onto the SU-8. We calculated the penetration depth of light into gold in the near-UV range to about $16 \mathrm{~nm}$ (for intensity). A $50-\mathrm{nm}-$ thick film therefore provides enough light attenuation for reliable masking. We note that gold and PDMS exhibit a low surface free energy leading to very poor adhesion between the two materials. The poor adhesion is crucial for the transfer printing process since it facilitates the release of the metal film from the stamp. The chromium film, on the other hand, is known to be more reactive promoting the adhesion of the mask to the SU-8 resist [3].

\section{Substrate}

The cantilever microsystem was fabricated on a silicon wafer easing the handling of the polymeric substrate. The wafer was first coated with a 50 -nm-thick fluorocarbon layer produced by plasma-polymerization of $\mathrm{C}_{4} \mathrm{~F}_{8}$ in a silicon dry etch device (ASE, STS-Surface Technology Systems plc, Newport, UK). This film reduces the adhesion of the polymer to the silicon due to a low surface free energy and facilitates the final release of the chips after the micromachining was completed. Next, a $30-\mu$ m-thick bottom layer of fully processed SU-8 (soft-baked, UV-exposed, post-exposure-baked) and a 50 - $\mu \mathrm{m}$-thick layer of nonpolymerized SU-8 (SU-8 50, MicroChem Corp., Newton, MA, USA) were deposited on top of the fluorocarbon film

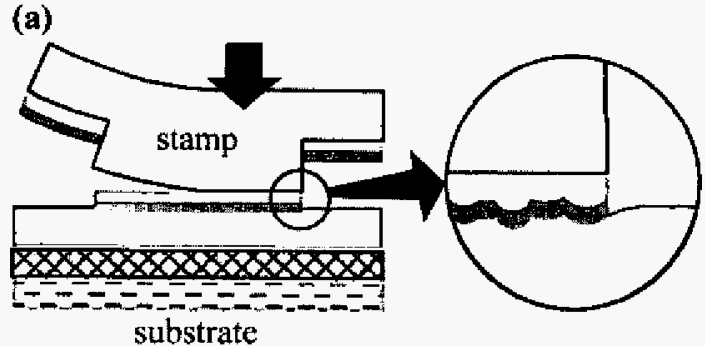

(b)

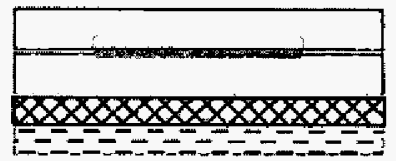

(c)

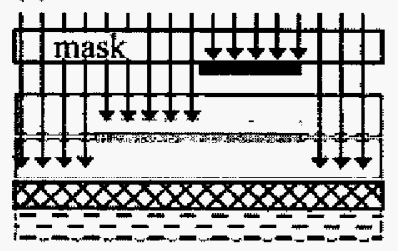

(d)

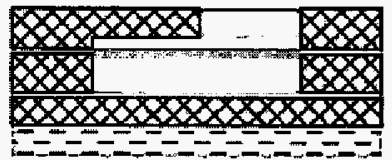

(e)

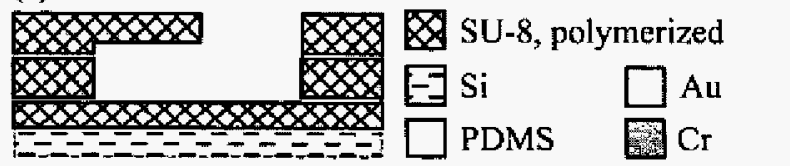

Figure 2: Process sequence for the fabrication of overhanging cantilevers in SU-8. (a) Contact printing of the mask on a SU-8 substrate composed of a polymerized bottom layer covered by a non-polymerized layer. The image shows a schematic cross-section of the stamp and substrate. (b) Spin-on of a thin SU-8 layer on top of the stamped mask. (c) Standard UV-light lithography to define the cantilever and the microchannel. The embedded mask protects the underlying resist from light exposure. (d) Polymerization of the UV-exposed resist by a post-exposture bake. (e) $S U-8$ development and mask etching to free the cantilever.

[Fig. 2(a)]. The non-polymerized SU-8 layer was only softbaked to evaporate the solvent. It was designated to host the microfluidic channel defined later after printing of the embedded mask.

\section{Contact Printing}

In the following, the overhanging cantilevers were fabricated on top of the non-polymerized SU-8 substrate. Therefore, the non-polymerized resist below the location where the beams were to be produced needed to be protected from UV-exposure by the gold/chromium mask. The mask was printed from the stamp onto the SU-8 at gentle pressure of 

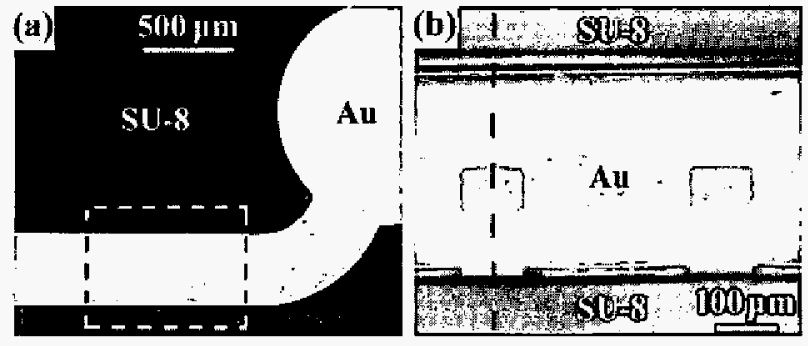

Figure 3: Optical light microscope images of the embedded mask at different process steps. (a) Mask on nonpolymerized $S U-8$ after transfer printing from the $P D M S$ stamp. (b) Embedded mask with cantilever structure on top after $U V$-exposure and post-exposwre bake. The image location corresponds to the marked area in (a). The crosssection of the substrate along the line drawn in (b) is shown schematically in Fig. 2(d).

about $1 \mathrm{MPa}$ for a period of $1 \mathrm{~min}$ [Fig. 2(a)]. During printing the $\mathrm{SU}-8$ substrate was heated to $47^{\circ} \mathrm{C}$, which is close to the glass transition temperature of the non-polymerized resist. Upon peeling the stamp off the SU- 8 the metal mask delaminated from the PDMS due to low adhesion and stuck to the SU-8 surface. Heating of the polymer proved to be an important parameter in the stamping process. Close to the glass transition temperature the viscosity of the nonpolymerized SU-8 decreases. The soft polymer adapted to the surface roughness of the metal mask, thus filling up the gaps in the corrugated surface [magnified inset in Fig. 2(a)]. This small polymer deformation on the nanometer-scale increased the contact area and improved the adhesion between the metal and the SU-8.

The printing process left a very flat metal mask on the SU-8 as shown in the light microscope image of Fig. 3(a) and the stylus profilometer image in Fig. 4. Apart from the elevation created by the metal mask printed onto the resist, Fig. 4 also reveals grooves in the SU-8 on both sides of the mask. These troughs correspond to plastic deformation of the non-polymerized SU-8 induced by the pressure applied during the stamping. The deformation of the resist is, however, in the range of only a few hundreds of nanometers. This low surface distortion was crucial to avoid reflow and thus wrinkling of the polymer and mask during subsequent baking and polymerization steps.

After mask printing the metal-patterned resist was covered with a thin SU-8 layer of several micrometer thickness [SU-8 2, MicroChem Corp., Newton, MA, USA, Fig. 2(b)]. This film served as the substrate in which the overhanging cantilevers were defined. The substrate was left for at least $2 \mathrm{~h}$ at a well-ventilated place to gently evaporate the solvent. This "soft bake" at room temperature minimized reflow of the non-polymerized material below the mask. Solvent from the freshly added SU-8 film can diffuse into the underlying non-polymerized layer reducing the material's viscosity and increasing its susceptibility to plastic deformation. The SU-8 was subsequently exposed to UV-light through a standard mask as shown in Fig. 2(c). During this

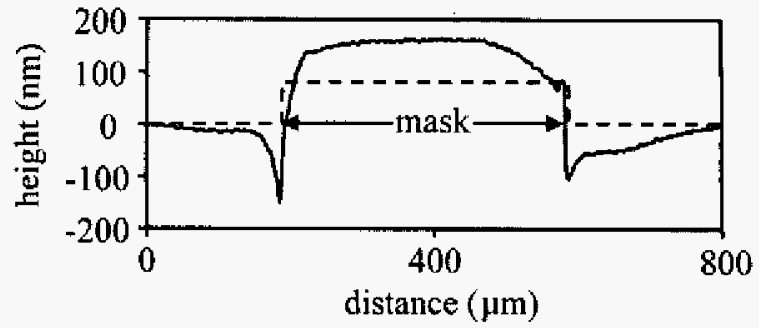

Figure 4: Surface profile across the mask printed onto nonpolymerized SU-8. The solid line shows the measured topography. The dashed line reflects the theoretical profile in absence of any surface deformation induced by printing.

lithography step the cantilever and microfluidic channel layout was projected onto the resist while the embedded shadow mask protected the resist underneath the cantilevers from UV-exposure. The exposed resist was polymerized by a post-exposure bake at $40^{\circ} \mathrm{C}$ for several hours [Fig. 2(d)]. The temperature during the baking was again set below the glass transition temperature of non-polymerized SU-8 to minimize material reflow. The light microscope image of Fig. 3(b) depicts the polymerized cantilever structure on top of the embedded mask after the post-exposure bake.

\section{Development}

The SU-8 substrate was subsequently developed to free the overhanging cantilevers [Fig. 2(e)]. To this end, the nonpolymerized SU-8 was dissolved in propylene-glycolmethyl-ether-acetate (PGMEA) and the gold/chromium mask etched away (GE 8148 and 1020 AC, Transene Company, Inc., Danvers, MA, USA). Note that the printed mask can also be used as a perfect screen for further lithography steps to, e.g., create piezoresistive wiring on top of the cantilevers [3].

\section{RESULTS AND DISCUSSION}

Figs. 5(a) and 5(b) show the cantilevers depicted in Fig. 3(b) after development. They are $100 \mu \mathrm{m}$ wide, overhanging a 50 - $\mu \mathrm{m}$-deep microfluidic channel for a length of $200 \mu \mathrm{m}$. The thickness of the cantilevers measures about $8 \mu \mathrm{m}$. In Figs. 5(c) and 5(d) cantilevers of $100 \mu \mathrm{m}$ width and $100 \mu \mathrm{m}$ length with a thickness of only $4 \mu \mathrm{m}$ are shown. Unlike the experiments performed by direct evaporation-deposition of embedded masks onto SU-8 [3] the beams fabricated via mask printing are perfectly flat. This result provides evidence of the importance of a 'soft' mask fabrication technique to achieve undeformed soft and sensitive overhanging membranes. To our knowledge these cantilevers are the thinnest overhanging SU-8 membranes published to date. The beam thickness compares well with the cantilever thickness achieved by the traditional chip bonding technique [3]. In principle, by diluting the purchased SU-8 resist, the fabrication of membranes down to $2 \mu \mathrm{m}$ thickness or less should be possible. Adding further solvent to the SU- 8 be- 


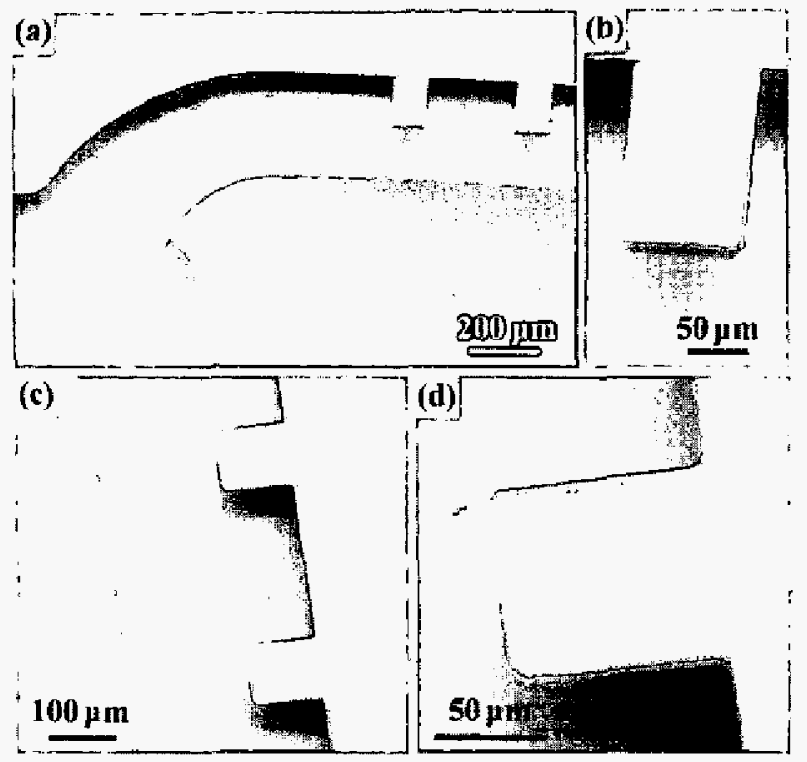

Figure 5: Electron microscope images of fully threedimensionally microfabricated cantilevers integrated in a microfluidic channel. The thickness of the beams in (a) and (b) measures $8 \mathrm{um}$, in (c) and (d) $4 \mathrm{\mu m}$. Note the flatness of the suspended structure.

fore processing allows for spin-coating of films significantly thinner than $4 \mu \mathrm{m}$ onto the resist.

\section{CONCLUSIONS}

A process based on contact printed masks for threedimensional microfabrication of highly sensitive cantilevers in SU-8 was demonstrated. The masks were transferred onto unexposed SU-8 from a flexible PDMS stamp. The mask transfer was mediated by a differential adhesion technique. Using a gold/chromium mask the gold surface facilitated delamination from the stamp while the chromium surface promoted the adhesion to the resist. Unlike evaporationdeposition, printing avoided high stress, vacuum, UV-light and electron bombardment, thus preventing resist wrinkling and pre-polymerization. The fabricated cantilevers have a very flat and well-defined surface emphasizing the importance of a 'soft' mask fabrication process to produce sensitive membranes. The microfluidic system fabricated here can be closed by applying a thick SU-8 layer over the substrate and priting a metal mask on top of it. This mask protects the resist underneath from UV-exposure during the definition of a channel lid in a further SU-8 layer spun over the metallization. Stacking of printed masks thus paves the way for complete three-dimensional micromachining on wafer scale of complex structures such as integrated cantile- vered probes, valves, pressure sensors and fine filters required for lab-on-a-chip devices.

\section{ACKNOWLEDGMENTS}

The authors thank P. A. Rasmussen and R. Marie from MIC for helpful discussions. A.B. acknowledges support from STVF, project $26-02-0280$ on polymer cantilevers. D.H. acknowledges support through a fellowship of the Swiss National Science Foundation.

\section{REFERENCES}

[1] L. J. Guérin, M. Bossel, D. Demierre, S. Calmes, P. Renaud, "Simple and low cost fabrication of embedded microchannels by using a new thick-film photoplastic", in Digest Tech. Papers Transducers ' 97 Conference, Chicago, USA, June 16-19, 1997, pp. 1419-1422.

[2] J. Thaysen, R. Marie, A. Boisen, "Cantilever-based biochemical sensor integrated in a microliquid handling system", in Digest Tech. Papers IEEE MEMS 2001 Conference, Interlaken, Switzerland, January 21-25, 2001, pp. 401-404.

[3] M. Calleja, P. Rasmussen, A. Johansson, A. Boisen, "Polymeric mechanical sensors with integrated readout in a microfluidic system", Proc. SPIE, vol, 5116, pp. 314-321, 2003.

[4] F. E. H. Tay, J. A. van Kan, F. Watt, W. O. Choong, "A novel micro-machining method for the fabrication of thick-film SU-8 embedded micro-channels", J. Micromech. Microeng., vol. 11, pp. 27-32, 2001.

[5] Y. J. Chuang, F. G. Tseng, J. H. Cheng, W. K. Lin, "A novel fabrication method of embedded micro-channels by using SU-8 thick-film photoresists", Sensors Actuators $A$, vol. 103, pp. 64-69, 2003.

[6] H. Yu, O. Balogun, B. Li, T. W. Murray, X. Zhang, "Building embedded microchannels using a single layered SU-8, and determining Young's modulus using a laser acoustic technique", J. Micromech. Microeng., vol. 14, pp. 1576-1584, 2004.

[7] W. H. Teh, U. Dürig, G. Salis, R. Harbers, U. Drechsler, R. F. Mahrt, C. G. Smith, J. H. Güntherodt, "SU-8 for real three-dimensional subdiffraction-limited two-photon microfabrication", Appl. Phys. Lett., vol. 84, pp. 4095-4097, 2004.

[8] H. A. Reed, C. E. White, V. Rao, S. A. Bidstrup Allen, C. L. Henderson, P. A. Kohl, "Fabrication of microchannels using polycarbonates as sacrificial materials", J. Micromech. Microeng., vol. 11, pp. 733-737, 2001.

[9] Y. L. Loo, R. L. Willett, K. W. Baldwin, J. A. Rogers, "Additive, nanoscale patterning of metal films with a stamp and a surface chemistry mediated transfer process: Applications in plastic electronics", Appl. Phys. Lett., vol. 81, pp. 562-564, 2002. 